\title{
Spin transport in diffusive superconductors
}

\author{
Jan Petter Morten, ${ }^{1}$ Arne Brataas, ${ }^{1}$ and Wolfgang Belzig ${ }^{2}$ \\ ${ }^{1}$ Department of Physics, Norwegian University of Science and Technology, 7491 Trondheim, Norway \\ ${ }^{2}$ Department of Physics and Astronomy, University of Basel, Klingelbergstrasse 82, 4056 Basel, Switzerland
}

(Received 8 September 2004; published 23 December 2004)

\begin{abstract}
We employ the Keldysh formalism in the quasiclassical approximation to study transport in a diffusive superconductor. The resulting $4 \times 4$ transport equations describe the flow of charge and energy as well as the corresponding flow of spin and spin energy. Spin-flip scattering due to magnetic impurities is included. We find that the spin-flip length is renormalized in the superconducting case and propose an experimental system to measure the spin accumulation in a superconductor.
\end{abstract}

DOI: 10.1103/PhysRevB.70.212508

PACS number(s): 74.25.Fy, 72.25.Hg, 72.25.Ba

Manipulation of spin-polarized currents can be used to study fundamental transport processes and might also provide new functionality in electronic devices. In ferromagnets $(\mathrm{F})$, the current is spin-polarized due to the spin-dependent density of states and the spin-dependent scattering potentials. In contrast, in $s$-wave superconductors (S), electrons with spin up and spin down and opposite momentum form Cooper pairs with no net spin. Nanoscale superconductors therefore display strikingly different properties when driven out of equilibrium by spin transport than by charge transport.

Most of the recent activities on the transport properties of F/S junctions have studied effects caused by the physical properties on the F side of the junction. The zero spin Cooper pairs prevent spin-polarized electrons to flow into S. Consequently, a spin-polarized current from $F$ injected into $S$ can result in nonequilibrium spin accumulation near the $\mathrm{F} / \mathrm{S}$ interface. The competition between electron-hole correlations and spin accumulation on the $\mathrm{F}$ side has recently attracted considerable interest. ${ }^{1}$ Possible influence of the ferromagnetic order parameter on the superconductor has received less attention. Singlet pairing does not allow a spin accumulation in the superconductor. Consequently, spin accumulation can reduce the superconducting gap and change the transport properties both for transport via quasiparticles and for the supercurrent. Experimentally, spin transport in diffusive $S$ has recently been studied. ${ }^{2}$ Here, the reduced quasiparticle penetration due to spin accumulation results in loss of spin memory which can be measured as a decreased magnetoresistance.

Although the theory of nonequilibrium superconductivity is widely used and developed, it has not been completely generalized to study spin transport. In this paper we thus use the Keldysh formalism and the quasiclassical approximation $^{3-5}$ to rigorously obtain a set of equations describing the transport of charge and energy in a diffusive weak coupling $\mathrm{S}$, as well as the transport of spin. This will describe the penetration of spins into $S$ and the associated suppression of the superconducting order parameter. Our description of the transport properties will be based on a 4 $\times 4$ matrix equation formalism to include spin accumulation as well as electron-hole correlations. Spin-flip scattering from magnetic impurities is included as the dominant spin relaxation process inside the superconductor. We find that the spin-flip length is renormalized in the BCS case, and propose an experimental system to measure the properties resulting from the superconducting correlations. Many, but not all, experimental systems involving spin transport in superconductors are in the elastic transport regime, ${ }^{6}$ which is considered here. Complementary studies based on the Boltzmann equation for spin-transport by quasiparticles in the inelastic transport regime have recently been published. ${ }^{7}$ Note that spin injection is qualitatively different in these opposite transport regimes due to the strong energy dependence of quasiparticle flow in superconductors. ${ }^{7}$

Let us now outline the derivation of our main results. We use natural units so that $\hbar=k_{B}=1$, and the electron charge is $e=-|e|$. To describe the out-of-equilibrium electron-hole correlations as well as spin accumulation, we define the Keldysh Green's function as

$$
\hat{G}_{i j}^{\mathrm{K}}(1,2)=\sum_{k}(-i)\left(\hat{\rho}_{3}\right)_{i k}\left\langle\left[(\psi(1))_{k},\left(\psi^{\dagger}(2)\right)_{j}\right]_{-}\right\rangle,
$$

where $\psi=\left[\psi_{\uparrow}, \psi_{\downarrow}, \psi_{\uparrow}^{\dagger}, \psi_{\downarrow}^{\dagger}\right]^{T}$ is a four-vector and $\psi^{\dagger}$ the corresponding adjoint vector. The matrix $\hat{\rho}_{3}$ is the third Pauli matrix generalized to $4 \times 4$ space, $\hat{\rho}_{3}=\operatorname{diag}(1,1,-1,-1)$. The coordinates are $1=\left(\mathbf{r}_{1}, t_{1}\right)$ and $2=\left(\mathbf{r}_{2}, t_{2}\right)$. Similarly, we define $4 \times 4$ retarded and advanced Green's functions $\left(\hat{G}^{\mathrm{R}}, \hat{G}^{\mathrm{A}}\right)$ in spin- and particle-hole space. $4 \times 4$ matrices are denoted by a "hat" superscript. A compact notation can be obtained by construction of an $8 \times 8$ matrix in the Keldysh space (denoted by a "check" superscript). ${ }^{5}$

The quasiclassical Green's function is defined by $\check{g}\left(\mathbf{R}, T, \mathbf{p}_{F}, E\right)=i / \pi \int d \xi_{p} \check{G}(\mathbf{R}, T, \mathbf{p}, E)$. This function is determined by the Eilenberger equation which in the mixed representation for a stationary state can be written

$$
\left[E \hat{\rho}_{3}+i \frac{\mathbf{p}}{m} \cdot \hat{\boldsymbol{d}}-e \phi \hat{1}-\hat{\Delta}-\check{\boldsymbol{\sigma}}, \check{g}\right]_{-}=0,
$$

where $\hat{\boldsymbol{\partial}}=\nabla \hat{1}-i e \mathbf{A} \hat{\rho}_{3}$ is the gauge invariant derivative, $\hat{1}$ is the $4 \times 4$ unit matrix, $\phi$ is the electromagnetic scalar potential, $\hat{\Delta}$ contains the superconducting gap, and $\check{\sigma}$ is the selfenergy due to elastic impurity scattering and spin-flip scattering by magnetic impurities in quasiclassical approximation. In the case of strong impurity scattering (dirty limit) transport is diffusive. Expansion of the quasi- 
classical Green's function in spherical harmonics then gives the Usadel equations. The symmetries and normalization of the Green's function allows for a parameterization of the quasiclassical, retarded component: ${ }^{4}$

$$
\hat{g}_{s}^{\mathrm{R}}=\left(\begin{array}{cc}
\overline{1} \cosh (\theta) & i \bar{\tau}_{2} \sinh (\theta) e^{i \chi} \\
i \bar{\tau}_{2} \sinh (\theta) e^{-i \chi} & -\overline{1} \cosh (\theta)
\end{array}\right),
$$

where $\overline{1}$ is the $2 \times 2$ unit matrix, $\bar{\tau}_{2}$ is the second Pauli matrix, and $\theta$ and $\chi$ are position and energy dependent functions. We assume colinear magnetizations along the $z$ axis and $s$-wave singlet superconducting state. We choose a gauge where the superconducting order parameter $\Delta$ is real and positive, and then the supercurrent is contained in the electromagnetic vector potential $\mathbf{A}$ and the chemical potential of the Cooper pairs is included in $\phi$. Inspection of the selfconsistency relation for $\Delta$ reveals that $\chi=0, \pi$ depending on the boundary conditions. This ansatz simplifies the calculations considerably. The advanced Green's function is related to the retarded through $\hat{g}^{\mathrm{A}}=-\left[\hat{\rho}_{3} \hat{g}^{\mathrm{R}} \hat{\rho}_{3}\right]^{\dagger}$. Because of normalization, the Keldysh Green's function can be expressed as $\hat{g}^{\mathrm{K}}=\hat{g}^{\mathrm{R}} \hat{h}-\hat{h} \hat{g}^{\mathrm{A}}$ where $\hat{h}$ is a diagonal distribution function matrix.

We will now consider a stationary state. A kinetic equation can be derived from the Usadel equations if we include Keldysh components. The important quantities are the physical particle and energy currents (including particles and holes), which we will denote by $\mathbf{j}_{\mathrm{T}}$ and $\mathbf{j}_{\mathrm{L}}$, respectively, with the corresponding distribution functions carrying the same indices, $h_{\mathrm{T}}$ and $h_{\mathrm{L}}{ }^{4}$ The physical spin current is denoted $\mathbf{j}_{\mathrm{TS}}$ and the spin energy current $\mathbf{j}_{\mathrm{LS}}$, with distribution functions $h_{\mathrm{TS}}$ and $h_{\mathrm{LS}}$. The spin-resolved distribution functions can be expressed by the particle distribution function as $h_{\mathrm{TS}(\mathrm{LS})}=$ $-\left[f_{\uparrow}(E)-f_{\downarrow}(E)\right] / 2-(+)\left[f_{\uparrow}(-E)-f_{\downarrow}(-E)\right] / 2$. The current components $\mathbf{j}_{\mathrm{T}}$, etc. are spectral quantities, and the total charge current is given as an integral $\mathbf{j}_{\text {charge }}(\mathbf{r}, t)$ $=|e| N_{0} \int_{-\infty}^{\infty} d E \mathbf{j}_{\mathrm{T}}(\mathbf{r}, t, E)$, and the spin current is obtained by a similar integral of $\mathbf{j}_{\mathrm{TS}}$. Energy current is given by $\mathbf{j}_{\text {energy }}(\mathbf{r}, t)=|e| N_{0} \int_{-\infty}^{\infty} d E E \mathbf{j}_{\mathrm{L}}(\mathbf{r}, t, E)$, and the difference in energy current carried by opposite spins by a similar integral of $\mathbf{j}_{\text {LS. }}$.

The equilibrium solutions for the distribution functions are $h_{\mathrm{L}, 0}=\tanh (\beta E / 2)$ and $h_{\mathrm{T}, 0}=h_{\mathrm{LS}, 0}=h_{\mathrm{TS}, 0}=0$. We derive kinetic equations and find:

$$
\begin{gathered}
\nabla \cdot \mathbf{j}_{\mathrm{L}}=0, \\
\nabla \cdot \mathbf{j}_{\mathrm{T}}=-2|\Delta| \alpha_{\mathrm{TT}} h_{\mathrm{T}}, \\
\nabla \cdot \mathbf{j}_{\mathrm{LS}}=-\left(2|\Delta| \alpha_{\mathrm{TT}}+\frac{1}{\tau_{\mathrm{sf}}} \alpha_{\mathrm{LSLS}}\right) h_{\mathrm{LS}}, \\
\nabla \cdot \mathbf{j}_{\mathrm{TS}}=-\frac{1}{\tau_{\mathrm{sf}}} \alpha_{\mathrm{TSTS}} h_{\mathrm{TS}} .
\end{gathered}
$$

The right-hand side terms represent renormalized scattering because of superconductivity:

$$
\alpha_{\mathrm{TT}}=\operatorname{Im}[\sinh (\theta)],
$$

$$
\begin{aligned}
& \alpha_{\mathrm{LSLS}}=\{\operatorname{Re}[\cosh (\theta)]\}^{2}-\{\operatorname{Im}[\sinh (\theta)]\}^{2}, \\
& \alpha_{\text {TSTS }}=\{\operatorname{Re}[\cosh (\theta)]\}^{2}+\{\operatorname{Re}[\sinh (\theta)]\}^{2} .
\end{aligned}
$$

The $|\Delta| \alpha_{\mathrm{TT}}$ terms on the right-hand side in Eq. (4) are due to conversion of quasiparticle current into supercurrent, and the $\alpha_{\mathrm{LSLS}} / \tau_{\mathrm{sf}}, \alpha_{\mathrm{TSTS}} / \tau_{\mathrm{sf}}$ terms are due to spin flips. The spin-flip time in the normal state is $\tau_{\mathrm{sf}}^{-1}=8 \pi n_{\mathrm{sf}} N_{0} S(S+1)\left|v_{\mathrm{sf}}\right|^{2} / 3$, where $n_{\mathrm{sf}}$ is the magnetic impurity density, $N_{0}$ the density of states at the Fermi level, $S$ the impurity spin quantum number, and $v_{\text {sf }}$ is the Fourier transformed spin-flip impurity potential. We assume isotropic scattering. Our definition of $\tau_{\mathrm{sf}}$ differs from the usual spin-flip lifetime by a renormalization factor $4 / 3$. This definition reproduces the diffusion equation with a spin-flip length $l_{\mathrm{sf}}^{(\mathrm{N})}=\sqrt{D \tau_{\mathrm{sf}}}$ in the normal state. Thus there is a difference between the spin-flip lifetime measured in, e.g., electron spin resonance and spin-flip transport time.

We introduce generalized energy-dependent diffusion coefficients

$$
\begin{aligned}
& D_{\mathrm{L}}=D\left(\{\operatorname{Re}[\cosh (\theta)]\}^{2}-\{\operatorname{Re}[\sinh (\theta)]\}^{2}\right), \\
& D_{\mathrm{T}}=D\left(\{\operatorname{Re}[\cosh (\theta)]\}^{2}+\{\operatorname{Im}[\sinh (\theta)]\}^{2}\right),
\end{aligned}
$$

where $D=\pi v_{F}^{2} / 3$ is the diffusion constant. The currents can then be expressed as

$$
\begin{gathered}
\mathbf{j}_{\mathrm{L}}=-D_{\mathrm{L}} \nabla h_{\mathrm{L}}+\operatorname{Im}\left\{\mathbf{j}_{E}\right\} h_{\mathrm{T}}, \\
\mathbf{j}_{\mathrm{T}}=-D_{\mathrm{T}} \nabla h_{\mathrm{T}}+\operatorname{Im}\left\{\mathbf{j}_{E}\right\} h_{\mathrm{L}}, \\
\mathbf{j}_{\mathrm{LS}}=-D_{\mathrm{T}} \nabla h_{\mathrm{LS}}+\operatorname{Im}\left\{\mathbf{j}_{E}\right\} h_{\mathrm{TS}}, \\
\mathbf{j}_{\mathrm{TS}}=-D_{\mathrm{L}} \nabla h_{\mathrm{TS}}+\operatorname{Im}\left\{\mathbf{j}_{E}\right\} h_{\mathrm{LS}},
\end{gathered}
$$

where we have defined the spectral supercurrent as $\mathbf{j}_{E}$ $=D(\nabla \chi-2 e \mathbf{A}) \sinh ^{2}(\theta)$. The self-consistency relation is

$$
\Delta(\mathbf{r})=-\frac{1}{2} \operatorname{sgn}\left(\Delta_{0}\right) N_{0} \lambda \int_{-\infty}^{\infty} d E \sinh (\theta) h_{\mathrm{L}},
$$

where the factor $\operatorname{sgn}\left(\Delta_{0}\right)$ is determined from the boundary condition to give the correct sign and $\lambda$ is the interaction parameter. The complex part of this equation is neglected as a consequence of charge conservation. ${ }^{8}$

The functions $\theta$ and $\chi$ are determined by the retarded components of the Usadel equation. We obtain

$$
\begin{gathered}
\nabla \cdot \mathbf{j}_{E}=0, \\
D\left(\nabla^{2} \theta-\frac{1}{2}(\nabla \chi-2 e \mathbf{A})^{2} \sinh (2 \theta)\right) \\
=-2 i E \sinh (\theta)-2 i \cosh (\theta)|\Delta|+\frac{3}{4} \frac{1}{\tau_{\mathrm{sf}}} \sinh (2 \theta),
\end{gathered}
$$

where Eq. (9) implies that the spectral supercurrent is conserved. In addition we have the following symmetry conditions, $\theta^{*}(-E)=-\theta(E), \chi^{*}(-E)=\chi(E)$. Equations (4) $-(10)$ determine all transport properties of $\mathrm{S}$. 


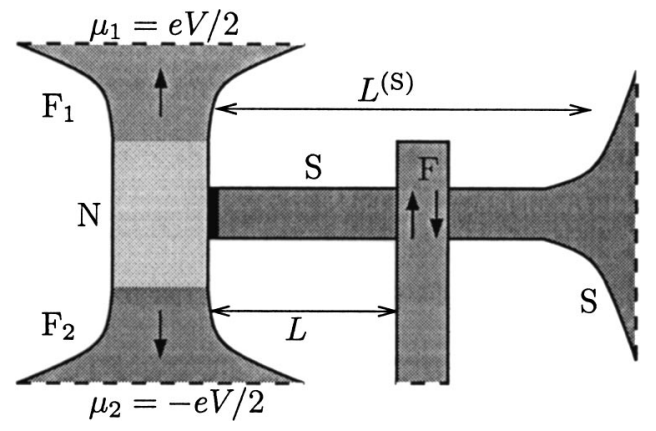

FIG. 1. Spin battery connected to a superconductor. The thick solid line indicates a tunnel barrier.

In general, in a hybrid $\mathrm{F} / \mathrm{S}$ system, the superconductor cannot be described as in terms of BCS formulas close to the F/S interface due to the proximity effect. Nevertheless, to gain insight into the physics implied by the abovementioned formulas let us now consider the limit of a homogeneous BCS superconductor, and select $\chi=0$. This is relevant for the proposed experiment below. For energies $|E|<|\Delta|, \alpha_{\mathrm{TT}}$ $=\Delta / \sqrt{\Delta^{2}-E^{2}}$ and the spin-flip renormalization factors are $\alpha_{\mathrm{TSTS}}=0, \alpha_{\mathrm{LSLS}}=-\Delta^{2} /\left(\Delta^{2}-E^{2}\right)$. The generalized diffusion constant $D_{\mathrm{L}}=0$ while $D_{\mathrm{T}}=D \Delta^{2} /\left(\Delta^{2}-E^{2}\right)$. From Eq. (7a) this means that there is no energy current carried by quasiparticles with energy $|E|<|\Delta|$. Gap scattering for quasiparticle energies below the superconducting gap corresponds to a transformation of the charge current $\left(\mathbf{j}_{\mathrm{T}}\right)$ into supercurrent. Such scattering is not possible for the physical spin current $\left(\mathbf{j}_{\text {TS }}\right)$. Consequently, in the absence of spin-flip scattering the quasiparticle spin current into the superconductor vanishes for $|E|<|\Delta|$ since $D_{\mathrm{L}}=0$ in Eq. (7d) and $\alpha_{\mathrm{TSTS}}=0$ in the kinetic Eq. (4d). Note that this result relies on the fact that there are different effective diffusion coefficients for charge current $\left(D_{\mathrm{T}}\right)$ and for spin current $\left(D_{\mathrm{L}}\right)$. We also observe that the term $\alpha_{\text {LSLS }}$ is negative below the gap, acting as a source of spin energy.

Above the gap $(|E|>|\Delta|)$ the factor $\alpha_{\mathrm{TT}}$ vanishes while $\alpha_{\mathrm{LSLS}}=E^{2} /\left(E^{2}-\Delta^{2}\right), \alpha_{\mathrm{TSTS}}=\left(E^{2}+\Delta^{2}\right) /\left(E^{2}-\Delta^{2}\right)$. For the generalized diffusion coefficients we find that $D_{\mathrm{L}}=D$ and $D_{\mathrm{T}}$ $=D E^{2} /\left(E^{2}-\Delta^{2}\right)$. Now consider the kinetic equations in the BCS case. A charge current carried by quasiparticles with energy $|E|>|\Delta|$ can propagate into $S$. For quasiparticles at $|E|>|\Delta|$ we see that there is no renormalization for the spinenergy diffusion length in Eq. (4c), whereas the spin diffusion length in Eq. (4d) has an energy dependent renormalization factor which diverges for energies $|E|=|\Delta|$ causing massive spin-flip scattering.

We will now apply this formalism to study spin diffusion, and demonstrate the significance of the renormalization of the spin diffusion length. Experimental studies of spin accumulation and spin injection has recently been performed ${ }^{9}$ in metallic spin valves. The spin accumulation in the physically different inelastic regime for a superconductor in this experimental system has also been calculated theoretically. ${ }^{7} \mathrm{We}$ will consider the simplified geometry shown in Fig. 1, where there is no charge transport in the superconductor, and calculate the spin-accumulation signal in the elastic regime. The $\mathrm{F}_{1} / \mathrm{N} / \mathrm{F}_{2}$ systems act as a spin battery which is connected via a tunnel junction to the superconductor. A voltage bias between $F_{1}$ and $F_{2}$ induces a spin accumulation that can flow into $\mathrm{S}$. The superconducting wire is connected to an $\mathrm{S}$ reservoir in equilibrium BCS state by a good metallic contact at distance $L^{(\mathrm{S})}$ from the N/S interface. On top of the $\mathrm{S}$ wire there is a ferromagnet connected by tunnel barrier which upon switching of the magnetization direction acts as a detector for the spin signal. Measurement of the relative voltage of this electrode between parallel and antiparallel (with respect to the top $\mathrm{F}$ reservoir) magnetization gives $\Delta \mu$ $=\mu^{(\mathrm{P})}-\mu^{(\mathrm{AP})}$ which describes the difference between electrochemical potential of spin-up and spin-down quasiparticles located a distance $L$ from the N/S interface. This quantity can be calculated $\Delta \mu=-\int_{-\infty}^{\infty} d E P^{(\mathrm{D})} h_{\mathrm{TS}}(L, E)$, where $P^{(\mathrm{D})}$ is the spin polarization of the tunnel barrier between $\mathrm{S}$ and the $\mathrm{F}$ detector. We assume a homogeneous order parameter and BCS spectral properties in the $\mathrm{S}$ wire since there are tunnel barriers between the $\mathrm{N}, \mathrm{F}$, and $\mathrm{S}$ elements and perturbation from current and spin-flip is weak.

We can express the difference between the spin-up and spin-down distribution functions in $\mathrm{N}$ close to $\mathrm{S}$ as $\Delta f^{(\mathrm{N})}$ $\equiv f_{\uparrow}^{(\mathrm{N})}-f_{\downarrow}^{(\mathrm{N})}=P^{(\mathrm{FN})}[f(E-e V / 2)-f(E+e V / 2)]$, where $P^{(\mathrm{FN})}$ $=\left(G_{\mathrm{maj}}-G_{\mathrm{min}}\right) /\left(G_{\mathrm{maj}}+G_{\mathrm{min}}\right)$ is the spin polarization between the $\mathrm{F}$ reservoirs and $\mathrm{N}, f(E \pm e V / 2)$ is the Fermi-Dirac distributions in the F reservoirs and $G_{\text {maj(min) }}$ is the conductance of majority (minority) spin electrons from ferromagnetic reservoir to the middle of $\mathrm{N}$. There is thus no charge current or supercurrent anywhere in S, however there may be a spin current. Equation (7d) states that there is no spin-current for energies below the gap, thus for these energies the N/S interface is effectively insulating. Since the $\mathrm{S}$ wire is connected to a reservoir in the other end for $|E|<\Delta$ the spin distribution function equals the equilibrium value $h_{\mathrm{TS}}=0$. We solve the TS kinetic Eq. (4d) for energies $|E|>\Delta$. This equation reduces to a diffusion equation with renormalized spinflip length $l_{\mathrm{sf}}^{(\mathrm{S})}(E)=l_{\mathrm{sf}} \sqrt{\left(E^{2}-\Delta^{2}\right) /\left(E^{2}+\Delta^{2}\right)}$, where $l_{\mathrm{sf}}=\sqrt{D \tau_{\mathrm{sf}}}$ is the normal state spin-flip length. The boundary condition at the $\mathrm{S}$ reservoir is that the distribution function attains the equilibrium value, and at the $\mathrm{S} / \mathrm{N}$ interface we match at each energy the tunnel spin current to the spin current inside $S$, $|e| N_{0} \mathbf{j}_{\text {TS }}$. We assume that $L^{(\mathrm{S})} / l_{\mathrm{sf}}^{(\mathrm{S})} \gg 1$ which is a relevant physical situation.

The position and energy dependent solution $h_{\mathrm{TS}}$ is substituted into the expression for the measured difference in electrochemical potential for parallel and antiparallel configuration, and we obtain

$$
\Delta \mu=2 P^{(\mathrm{D})} \int_{\Delta}^{\infty} d E \Delta f^{(\mathrm{N})} e^{-L / l_{\mathrm{sf}}^{(\mathrm{S})}} \frac{R_{\mathrm{sf}}^{(\mathrm{S})}}{R_{\mathrm{sf}}^{(\mathrm{S})}+R^{(\mathrm{I})}},
$$

where $R^{(\mathrm{I})}(E)=1 /\left[|T|^{2} N_{\mathrm{BCS}}(E) N_{0}\right]$ is the resistance of the $\mathrm{N} / \mathrm{S}$ tunnel barrier, $|T|$ is the tunneling matrix element, $N_{\mathrm{BCS}}(E)$ is the BCS density of states, $R_{\mathrm{sf}}^{(\mathrm{S})}(E)=l_{\mathrm{sf}}^{(\mathrm{S})}(E) \rho / A$ is the resistance of the $\mathrm{S}$ wire within a spin-flip length and $\rho$ is the resistivity of the material in $\mathrm{S}$ when in the normal state $\left(T>T_{c}\right)$. This result can be understood as follows. The spinaccumulation close to the tunnel interface is exponentially attenuated by spin-flip scattering in $\mathrm{S}$. The spin signal is also 
decreased by the tunnel resistance, and since spin current is strongly suppressed for energies $|E|<\Delta$ only quasiparticles with energies higher than the gap contribute. The effective total resistance is a series of the tunnel interface resistance with the resistance of S within one spin-flip length.

We will now consider some simplified limits for the quantity $\Delta \mu$ defined above. In the normal state where $\Delta \rightarrow 0$ we find that $\Delta \mu / e V=2 \mathrm{P}^{(\mathrm{D})} P^{(\mathrm{FN})} R_{\mathrm{sf}}^{(\mathrm{S})} \exp \left(-L / l_{\mathrm{sf}}\right) /\left(R_{\mathrm{sf}}^{(\mathrm{S})}+R^{(\mathrm{I})}\right)$ where $R_{\mathrm{sf}}^{(\mathrm{S})}$ and $R^{(\mathrm{I})}$ assume their normal state (energy independent) values. At $k_{B} T \ll \Delta$ the signal measured by $\Delta \mu$ vanishes when the bias is lower than the energy gap $\mathrm{eV}<\Delta$ since spin current is suppressed for quasiparticles below the gap. For higher bias, $e V>\Delta$, and at zero temperature when the bulk resistance dominates, $R_{\mathrm{sf}}^{(\mathrm{S})} \gg R^{(\mathrm{I})}$, an approximate solution is $\quad \Delta \mu=2 P^{(\mathrm{D})} P^{(\mathrm{FN})} \Delta e^{-L / l_{\mathrm{sf}}}\left\{e^{-L r^{2} / 2 l_{\mathrm{sf}} / r-e^{-L / 2 l_{\mathrm{sf}}}}\right.$ $\left.+\sqrt{\pi L / 2 l_{\mathrm{sf}}}\left(\operatorname{erf}\left[r \sqrt{L / 2 l_{\mathrm{sf}}}\right]-\operatorname{erf}\left[\sqrt{L / 2 l_{s f}}\right]\right)\right\}$, where $r=2 \Delta / e V$. In this case the relation between the energy gap and the bias determines the magnitude of the spin signal, and the exponential decrease of the signal.

The temperature dependence of $\Delta \mu$ in the general case is given by a decrease from a constant value above $T_{c}$ as the temperature approaches zero. An example of this behavior is shown in Fig. 2. Here we have used the approximate temperature dependence $\Delta=1.76 T_{c} \tanh \left(1.74 \sqrt{T_{c} / T-1}\right)$. Our calculations show that the spin signal decreases due to superconducting correlations. For a large energy gap the spin accumulation vanishes completely at low temperatures. These effects can be explained by suppressed subgap spin current and massive spin-flip at energies close to the gap because of the superconducting correlations.

In conclusion, we have presented a formalism to describe elastic spin transport in superconductors with spin-flip scat-

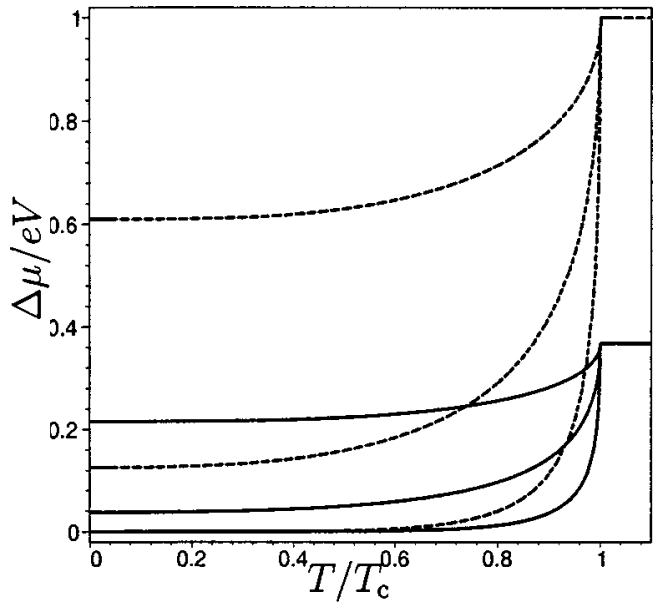

FIG. 2. Temperature dependence of $\Delta \mu / e V$. We use $R_{\mathrm{sf}}^{(\mathrm{S})}=R^{(\mathrm{I})}$ in the normal state. For the dotted lines $L / l_{\mathrm{sf}}=6$, and for the solid lines $L / l_{\mathrm{sf}}=7$. The bias $e V$ is $0.1 \Delta(T=0), 3 \Delta(T=0), 10 \Delta(T=0)$ for the lower curve to the higher curve, respectively.

tering. We find different effective diffusion coefficients for charge- and spin-current. The spin-flip length is renormalized in the superconducting case, and at energies close to the gap there is massive spin-flip. As an illustration we compute the difference in electrochemical potential due to spinaccumulation in an experiment sensitive to the renormalization of spin-flip length.

This work was supported in part by the Research Council of Norway, NANOMAT Grants No. 158518/431 and 158547/431, RTN Spintronics, the Swiss NSF and the NCCR Nanoscience.
${ }^{1}$ V. T. Petrashov, I. A. Sosnin, I. Cox, A. Parsons, and C. Troadec, Phys. Rev. Lett. 83, 3281 (1999); M. Giroud, H. Courtois, K. Hasselbach, D. Mailly, and B. Pannetier, Phys. Rev. B 58, R11872 (1998); V. I. Fal'ko, C. J. Lambert, and A. F. Volkov, Pis'ma Zh. Eksp. Teor. Fiz. 69, 497 (1999), JETP Lett. 69, 532 (1999); F. J. Jedema, B. J. van Wees, B. H. Hoving, A. Filip, and T. M. Klapwijk, Phys. Rev. B 60, 16549 (1999); D. HuertasHernando, Yu. V. Nazarov, and W. Belzig, Phys. Rev. Lett. 88, 047003 (2002).

${ }^{2}$ J. Y. Gu, J. A. Caballero, R. D. Slater, R. Loloee, and W. P. Pratt, Jr., Phys. Rev. B 66, 140507(R) (2002).

${ }^{3}$ N. Kopnin, Theory of Nonequilibrium Superconductivity (Oxford Science, London, 2001).
${ }^{4}$ W. Belzig, F. K. Wilhelm, C. Bruder, G. Schön, and A. D. Zaikin, Superlattices Microstruct. 25, 1251 (1999).

${ }^{5}$ J. Rammer and H. Smith, Rev. Mod. Phys. 58, 323 (1986).

${ }^{6}$ Y. Tserkovnyak and A. Brataas, Phys. Rev. B 65, 094517 (2002).

${ }^{7}$ T. Yamashita, S. Takahashi, H. Imamura, and S. Maekawa, Phys. Rev. B 65, 172509 (2002); S. Takahashi and S. Maekawa, ibid. 67, 052409 (2003).

${ }^{8}$ A. Schmid, of NATO Advanced Study Institute Series B, (1981), Vol. 65, pp. 423-480.

${ }^{9}$ M. Johnson and R. H. Silsbee, Phys. Rev. Lett. 55, 1790 (1985); F. J. Jedema, A. Filip, and B. J. van Wees, Nature (London) 410 , 345 (2001). 